\title{
Therapiestandards bei CED werden meist nicht umgesetzt
}

\begin{abstract}
Die Häufigkeit chronisch entzündlicher Darmerkrankungen nimmt offenbar umweltbedingt - weiter zu. Therapiestandards werden überwiegend nicht befolgt.
\end{abstract}

Die in den vergangenen 50 Jahren stark gestiegene Häufigkeit chronisch entzündlicher Darmerkrankungen (CED) ist nach Auffassung von Gastroenterologen bevorzugt auf Umweltfaktoren zurückzuführen. Es sei keinesfalls ein stabiles Niveau erreicht worden, wie teils berichtet werde, sagte Prof. Dr. Gerhard Rogler, Zürich. Vielmehr nehme die Zahl der Neuerkrankungen weiter zu, auch bei Kindern. In Deutschland geht man derzeit von einer Inzidenz für Morbus Crohn von 6 - 7/100.000 aus, für Colitis ulcerosa von $4-6 / 100.000$.
Als eine Ursache dieser Entwicklung wird die Luftverschmutzung im Zuge der Industrialisierung im 20. Jahrhundert diskutiert. Gesicherte Umweltfaktoren, die den Verlauf der Erkrankung beeinflussen und die Schübe triggern können, sind nach Rogler unter anderem Vitamin-D-Mangel, Gebrauch nichtsteroidaler Antirheumatika (NSAR) an mehr als 15 Tagen pro Monat, psychische Belastungen sowie orale Kontrazeptiva. Experimentelle Untersuchungen bei Tieren sowie bei gesunden Probanden in der Schweiz belegen darüber hinaus, dass lange Flugreisen oder der Aufenthalt in Höhen von über 2000 Metern über dem Meeresspiegel Hypoxie-bedingt CEDSchübe triggern können.

Die pragmatische Empfehlung Roglers ist zum einen das Meiden bekannter Trig- ger, soweit möglich, sowie gegebenenfalls die vorbeugende Behandlung. „Ein Viertel der CED-Schübe wären vermeidbar, wenn auf NSAR verzichet würde“, erklärte er. Hohe Vitamin-D-Spiegel schützen Crohnund Colitis-ulcerosa-Patienten vor Schüben. Vor Flugreisen könne die MesalazinDosis (z.B. Salofalk ${ }^{\circledast}$ ) erhöht bzw. Budesonid verordnet werden. Die Therapiequalität bei CED müsse deutlich verbessert werden, fordern Experten. Im Alltag erhalten weniger als 25\% der CED-Patienten die optimale Behandlung, wie z.B. die Kombination aus oraler und rektaler Mesalazintherapie, entsprechend der in Leitlinien definierten Standards.

(TM)

Symposium 205 der Falk Foundation e.V. "New Treatment Targets in Gut and Liver Diseases", Oktober 2016 in Luzern

\section{Hoffnung für PBC-Patienten}

\section{Neues Medikament schützt Leber und Galle}

Die europäische Zulassungsbehörde EMA hat im Oktober 2016 die vorläufige Zulassung von Obeticholsäure für schwer behandelbare Patienten mit primär biliärer Cholangitis (PBC) empfohlen. Es bestehe ein hoher Bedarf für PBC-Patienten, die unzureichend auf das bislang einzige zugelassene Medikament Ursodesoxycholsäure (UDCA) ansprechen, hieß es.

\begin{abstract}
Obeticholsäure, das in Kombination mit UDCA (z.B. Ursofalk ${ }^{\circledR}$ ) oder bei UDCAUnverträglichkeit allein angewendet werden soll, aktiviert den Farnesoid-X-Rezeptor, so dass die Gallensäureproduktion der Leber vermindert wird.

UDCA dagegen führt zu einem relativen Austausch toxischer Gallensäuren gegen die hydrophile, untoxische UDCA. Vermutlich verstärke sie außerdem die Sekretion von Bikarbonat, welches die Cholangiozyten vor den aggressiven Gal-
\end{abstract}

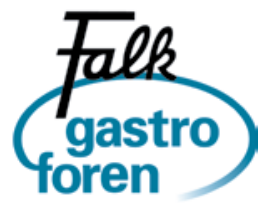

Besuchen Sie das nächste Falk Gastro Forum „Praktische Gastroenterologie 2017 - aktuelle Leitlinien und Therapiestandards" am Samstag, den 21. Januar 2017 in Karlsruhe; Infos: www.drfalkpharma.de/veranstaltungen lensäuren schützt, erklärte Prof. Dr. Ulrich Beuers, Amsterdam. Auf diese Weise werde die Fibrosierung der Leber verlangsamt und die Prognose der Patienten verbessert.

Die potenziell lebensbedrohliche PBC führt zur allmählichen Zerstörung der kleinen Gallenwege in der Leber. Hätten früher fast alle diese Patienten eine Leberzirrhose entwickelt, treffe dies seit Einführung von UDCA für ca. 50\% der Patienten nicht mehr zu, so Beuers. Zwei Drittel der Patienten hätten eine normale Lebenserwartung, nur noch selten sind Lebertransplantationen erforderlich. „Das ist ein großer Erfolg.“

(TM) 